is a CAL program to help

\title{
CA PTEX
}

students of Phonetics to learn

phonetic transcription

The program

- sets an exercise

- corrects and scores the student's work

- gives help and comments on errors

- keeps a log of activity, which an instructor can inspect

- provides a hard copy of the student's work

In addition, CAPTEX provides facilities for the instructor to

- create new phonetic symbols

- create new exercises

- work with different accents of English, or with different languages

(British English (RP) and American English are supplied ready to use)

Requirements: any PC (IBM or compatible) with colour graphics screen

Price: $£ 50$ includes Student Disk, Instructor Disk, Manual, Keyboard Overlay

Available from:

Oxford University Phonetics Laboratory

41 Wellington Square, Oxford OX1 2JF, U.K.

Tel. 0865-270444

${ }^{\star \star \star}$ COMING SOON: CAPTAPE (Computerized learning of phonetic transcription from TAPE-RECORDED speech) 
Make the most of your BBC micro

PHONETICS IN VIEW gives IPA phonetic symbols for use with the Acorn VIEW word-processor and Epson printers.

Full range of symbols and diacritics including

[E

[E

on an easily edited character file.

Special Printer Driver allows printer commands to be accessed by VIEW highlights or embedded directly in text, allowing changes of print size and density,

cuporecripting or eubecripting

ad-hoc character re-definitions (@ now prints $\checkmark$ ).

Printer driver, character files and manual $£ 20$

LAB Computer-assisted exercises in matching phonetic symbols and labels. Different datafiles available for wide range of symbol-label pairs including sets for standard text and reference books ( $D$ Connor, Gimson, EPD). New files can be made up to order, or created using utilities supplied with package.

2 Disks, with master program, datafiles, utilities prograns and manual $£ 20$

SURVEY Programs for creation and random or constant order recording and playback of wordlists, using Tandberg TCCR530 tape-recorder f10

Details from Stephen Parkinson, Department of Linguistics University of Aberdeen, King's Callege,

Aberdeen AB9 2UB, tel. 0224-272165

(from 1.10.88, Linacre College, Oxford) 


\section{PA fonts for the Macintosh ${ }^{\mathrm{TM}}$}

From: Linguists' Software 106R Highland St. South Hamilton, MA 01982 (717) 468-3037

\section{MacPhonetics}

3 fonts in many sizes: The complete IPA (International Phonetic Alphabet including all of its diacritical marks) SIL (Summer Institute of Linguistics symbol set), and MacTransliterator with 75 languages in one font!

Overstrike keys with automatic non-deleting backspacing allow extremely fast insertion of accents, umlauts, and a wide variety of other diacritical marks ( 37 in IPA, 53 in SIL, 50 in Roman=Mactransliterator). All of these can be joined with any letter, lower or upper case. A mini space bar which advances the insertion point a single pixel facilitates fine tuning of diacritical marks. Vertical bar for continuous vertical columns any number of lines in length.

High quality printing. The IPA font is selected directly from the font menu of MacWrite ${ }^{\text {Tx }}$ or Microsoft $(B)$ Word for automatic footnoting. Instantly transforms your keyboard to and from IPA, SIL and Roman. Style variations include bold, italic, underline, outline, shadow, superscript, and subscript. Not copy-protected. User's Manual and laminated keyboard layout sheet. (C) 1985 P. B. Payne

\section{fortfrcoming:}

\section{$\operatorname{LaserIPA}^{\mathrm{TM}}$}

$\$ 99.95$

Two International Phonetic Alphabet PostScript LaserWriter fonts with corresponding bit map screen and Imagewriter fonts: IpaTimes, with Times as the a-z font, authorized by Adobe Systems, and LaserIPAplus, a non-serifed Helvetica style font. Contains all of the Intemational Phonetic Alphabet symbols and all of the IPA diacritical marks with automatic non-deleting backspacing so they can overstrike any symbol and in any combination with other overstrikes. Complete with the new LaserWriter driver and LaserPrep software, User's Manual and laminated keyboard layout sheet. Prints all sizes from 4 point to 127 point. Not copy protected.

LaserWriter sample:

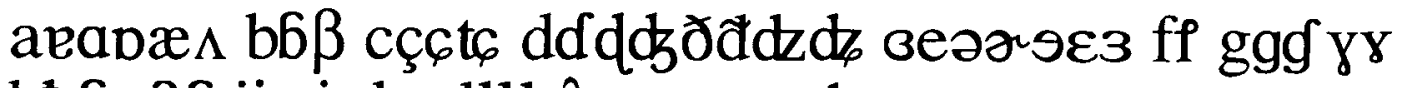

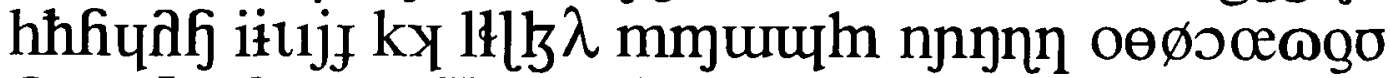

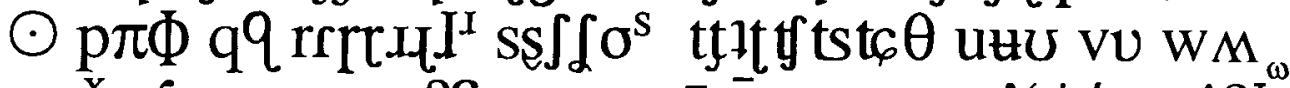

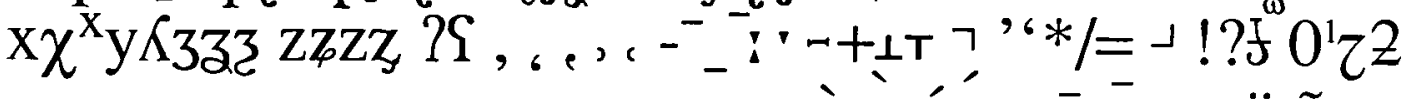

- o^ 


\section{AUDLAB \\ AN INTERACTIVE SPEECH AND SIGNAL ANALYSIS SYSTEM}

AUDLAB is a new speech and signal analysis package from the Centre for Speech Technology Research, University of Edinburgh. It has been designed as a nexible, sophisticated, yet easy to use system for a wide variety of applications, from supporting the teaching of introductory courses in speech acoustics to providing analytic tools for advanced research projects. The package is already running successfully at sites throughout the UK, Europe and the USA.

\section{AUDLAB FEATURES}

* Menu-drive operation

* Use of array processor:- for speed of execution.

- Powerful graphical display routines:- colour or black/white

- Multiple display windows:- it is possible to inspect simultaneously the results of a number of speech processing algorithms time-aligned against both the time-amplitude and spectrographic versions of the speech samples.

* User-friendly interface:- a powerful executive program provides a user-friendly interface for easy interactive signal processing.

* User added code:- it is easy for users to add their own algorithms to the speech and signal processing libraries.

\section{AUDLAB FACILITIES}

* Record and playback.

- Spectrographic displays:- both conventional and contrast-enhanced.

* Digital signal processing:- includes FFT, LPC, correlation, cepstral, pole zero modelling, digital filtering and filter design

- Speech analysis algorithms:including pitch-tracking, formant-tracking and parameters supporting voicing decisions.

* Waveform editing:- cut, paste and splice operations.

* Transcription and annotation facilities:- allowing time-aligned transcriptions of data files.

* An interpreter:- produces new parameters based on mathematical and/or logical relationships between existing parameters.

For further information contact:- The Centre for Speech Technology Research, University of Edinburgh, 80, South Bridge, Edinburgh EH1 1HN, Scotland. Tel: $031-225-8883$ 


\section{Coming soon...}

\section{THE DELTA SYSTEM, VERSION 2}

designed to let you conveniently express and test phonological, phonetic, and text-to-speech rules

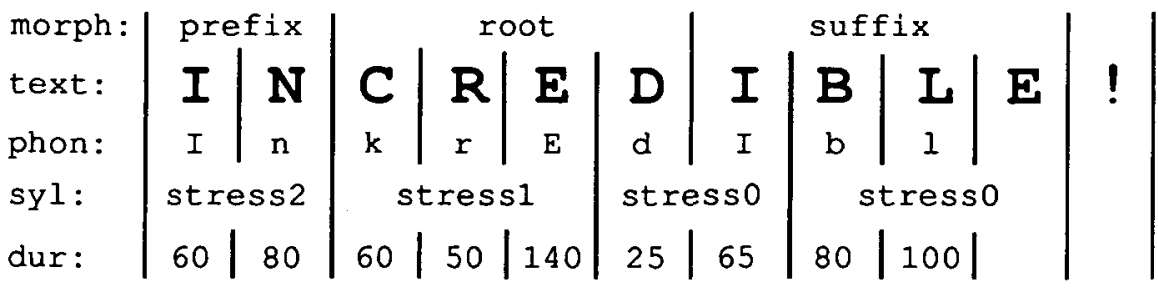

- Programming language for building and testing "multi-stream" utterance representations like the above, and synthesizing speech from them.

- Source-level debugger for tracing, testing, and modifying program (rule) execution.

- Flexible dictionary facilities.

- Can accomodate any phonological or phonetic model (e.g., multi-tiered or linear, articulatory or acoustic).

- Compiles Delta rules into C programming language statements, and lets users freely intermingle Delta and $C$ statements.

- Requires no previous knowledge of computer programming.

- Runs on most computers with $512 \mathrm{~K}$ of memory and standard $\mathrm{C}$ compiler.

Version 1, a subset of Version 2 available now

For more information, contact

Sue Hertz

ELOQUENT TECHNOLOGY

24 Highgate Circle

lthaca, NY 14850, USA

(607) 257-6829

Other Eloquent Technology products include Speech synthesis rules for English and Japanese 


\section{Journal of the International Phonetic Association}

\section{NOTES FOR CONTRIBUTORS}

Policy - The Journal of the International Phonetic Association (formerly entitled Le Maitre Phonétique), is concerned with all aspects of the theory, description and use of phonetics and phonology. Contributions are subject to review, and to the Editor's final decision as to publication. The official language of the Journal is English, though contributions in other languages of wide currency will be published as the Editor thinks fit.

Style - Contributors are encouraged to submit their text, if possible, on Macintosh floppy disc ( $800 \mathrm{~Kb}$ or $400 \mathrm{~Kb})$, in Helvetica font. The Journal is set using MacAuthor, which is compatible with MacWrite, but not with Microsoft Word. Documents formatted in Word should be transferred in Text Only form. In other circumstances, contributions should be submitted in typewritten form, in double spacing with wide margins, on one side of the sheet only, on quarto or A4 paper. The style conventions of the Journal should be followed wherever possible, and the Editor reserves the right to alter contributions so that they conform to house style. Footnotes should be avoided as far as possible; where essential they should be indicated in superscript consecutively throughout the text, and collected as a separate sheet at the end of the manuscript. References are to be made IN THE TEXT by giving in parentheses the name of the author and the year of publication, and where relevant the page(s) referred to: e.g. '... (Jones, 1963) ...', or '... Jones (1983: 453) .... All works referred to should be listed at the end of the article, following the conventions used in this number of the Journal. Phonetic transcriptions should make use of the symbols and conventions of the Association's alphabet. The set of phonetic symbols used in the Journal is available as IPA Plus from UCLA Phonetics Laboratory, free of charge except for the media cost. Phonemic symbols should be enclosed in slants, but allophonic or general-phonetic symbols in square brackets, e.g. / tel /, [ $\left.\mathrm{t}^{\mathrm{h}} \mathrm{kt}\right],[\mathrm{m}]$. Figures should be drawn to a standard which . will allow them to be reproduced clearly in photo-reduction.

Proofs and offprints . - Two copies of proofs will be sent to the author (or their nominee), who will be expected to correct them and return them to the Editor, by airmail where appropriate, within ten days of receipt. Upon publication, twenty five copies of a contribution will be supplied to the author free of charge; further offprints may be purchased at cost. 